\title{
Nevoid hyperkeratosis of the nipple mimicking a seborreic keratosis
}

\begin{abstract}
Nevoid hyperkeratosis of the nipple and/or areola (NHNA) is an uncommon skin disease with an unknown etiology, which occurs predominantly in women of childbearing age. Initially described in the medical literature by Tauber in 1923 as a benign dermatologic change in the nipple and areola. Clinically characterized by brown and verrucous thickening which are often bilateral. The differential diagnosis of NHNA includes Paget's disease, basal cell carcinoma, seborrheic keratosis, melanoma, erosive adenomatosis, and hyperkeratosis secondary to prolonged friction, so it's always considered as diagnosis of exclusion which requires histologic confirmation. The therapeutic management is generally justified by the anesthetic discomfort of the patient. Various therapeutic modalities have been used with varying success. We report a rare case of unilateral Nevoid hyperkeratosis of the nipple clinically and dermoscopically identical to a seborreic keratosis.
\end{abstract}

Keywords: dermoscopy, nevoid hyperkeratosis of the nipple, seborreic keratosis, histology
Volume 2 Issue 6 - 2018

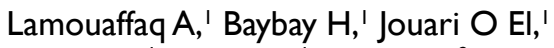
Senhaji G,' Gallouj S,' Rimani M,' Mernissi FZ'

'Department of Dermatology, University Hospital Hassan II, Morocco

${ }^{2}$ Hassan Center of anatomo-pathology, Morocco

Correspondence: Amina Lamouaffaq, Department of Dermatology, University Hospital Hassan II Fes, Route Sidi Harazem, Fes, Morocco, Tel 002I 26676132 13,

Email a.lamowaffaq@gmail.com

Received: October 09, 2018 | Published: November 05, 2018

\section{Introduction}

Nevoid hyperkeratosis of the nipple and/or areola (NHNA) is an uncommon skin disease with an unknown etiology in which the nipple and/or areola are covered with diffuse, hyperpigmented, verrucous, and asymptomatic plaques ${ }^{1,2}$ most predominant in the female gender between puberty and the third decade. ${ }^{3}$ We report a rare case of unilateral lesion on the nipple clinically and dermoscopically identical to a seborreic keratosis. Nevertheless, histopathologic examination suggested the diagnosis of NHNA.

\section{Observation}

A 22-years-old unmarried woman, presented with a painless, nonitchy, brownish lesion on here right nipple of 2years duration. On examination, there was a well-defined pigmented plaque, measured $5 \mathrm{~mm}$ on the major axis, with a keratotic surface at the infero-external part of the right nipple, respecting the areola, without local infiltration or erosion or breast flow or inflammatory signs (Figures 1). The contralateral nipple was normal. The dermoscopy demonstrated the presence of a brownish, homogeneous pigmentation and pseudocysts, suggesting seborrheic keratosis (Figures 2). There were also multiple well-defined pigmented plaques with a smooth surface, at the trunk, evoking seborrheic keratosis. Breast examination and ganglionic areas were normal. Histology objectified orthokeratotic hyerkeratosis, with a papillomatous epidermal lining and elongated filiform and anastomotic epidermal crests, thus confirming the dianostic (Figures 3) (Figure 4). The patient underwent complete excision of the lesion without recurrence after 2 years.

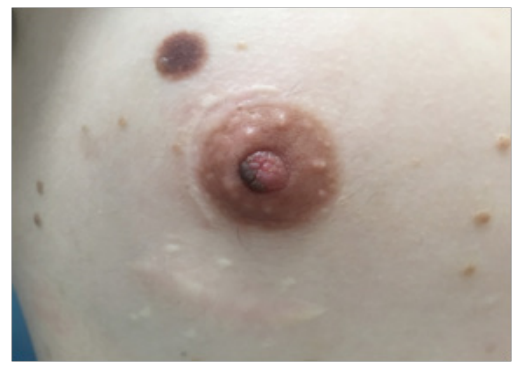

Figure I Pigmented plaque, measured $5 \mathrm{~mm}$ on the major axis, with a keratotic surface at the infero-external part of the right nipple, respecting the areola.

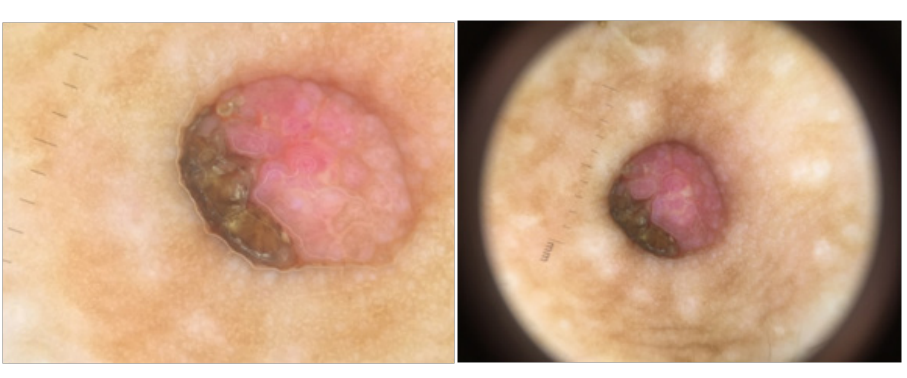

Figures 2 Presence of a brownish, homogeneous pigmentation and pseudocysts in dermoscopy examination.

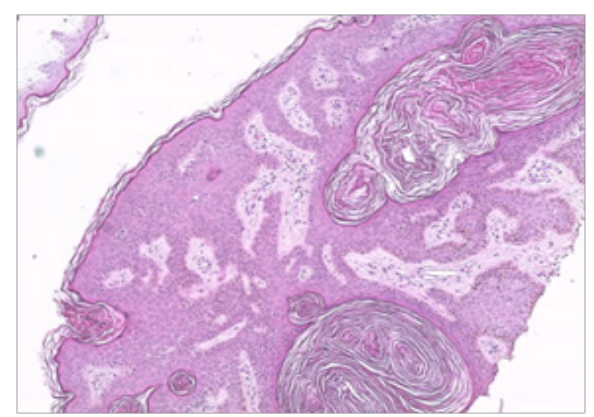

Figure $3 \mathrm{H}$ \& E stain G x 100 -> Irregular papillomatosis with elongate and anastomotic epidermal ridges + Dermis fibrosis + hyperkeratosis.

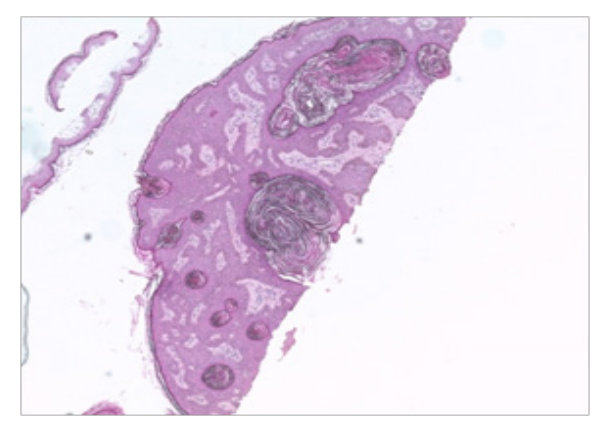

Figure $4 \mathrm{H}$ \& E stain G $\times$ 50-> Irregular papillomatosis with elongated and anastomotic epidermal ridges + dermal fibrosis + hyperkeratosis. 


\section{Discussion}

NHNA is a rare and benign skin disease, which occurs predominantly in women of child-bearing age, especially during the second and the third decades of life. ${ }^{4}$ It's first described in the medical literature by Tauber in 1923 as a benign dermatologic change in the nipple and areola, it may involve the nipple, the areola or both. ${ }^{5}$ Approximately 50 cases have been reported in the literature. ${ }^{2}$ Its etiology remains poorly understood and thus its treatment is empirical with an unpredictable therapeutic outcome. It characterized by brown and verrucous thickening of the nipple and/or areola, the lesions are often bilateral affecting the areola and nipple in $72 \%$ of cases, and has been classified into 3types by Levy-Frenckel in 1938, first, Type 1 who that associated with an epidermal nevus. Then, Type 2 who that associated with various dermatoses such as acanthosis nigricans, Darier disease, chronic eczema, and cutaneous T-cell lymphoma and finally, Type 3 that isolated form with unknown etiology. ${ }^{1,6}$ There are doubts on whether nevoid hyperkeratosis of the nipple and areola is a distinct entity or a clinical presentation of various dermatoses. Type III HNA is seen in females at puberty, those of childbearing age, pregnancy and also in males receiving hormonal therapy for prostate cancer. It may also be seen in females and males without the above mentioned conditions. Therefore, hormonal etiology could be discussed but not yet proven. ${ }^{7}$ The differential diagnosis of NHNA includes Paget's disease, basal cell carcinoma, seborrheic keratosis, melanoma, erosive adenomatosis, and hyperkeratosis secondary to prolonged friction, ${ }^{8}$ so it's always considered as diagnosis of exclusion. ${ }^{1}$ In our case, the presence of clinical and dermoscopical caracteristics of seborrheic keratoses may confuse the diagnosis which must be confirmed by histology. Histologically, the presence of hyperkeratosis, acanthosis and papillomatosis confirm the diagnostic of NHNA. ${ }^{9}$ And considering clinical and histopathologic findings, our patient's disease is most consistent with NHNA type 3. The therapeutic management is generally justified by the anesthetic discomfort of the patient. Various therapeutic modalities have been used with varying success, ${ }^{10}$ Keratolytic agents, topical corticosteroids, cryotherapy, surgical removal of the artery and reconstruction with a skin graft, ablation with a radiofrequency surgical unit, and treatment with carbon dioxide laser and topical calcipotriol have all been tried with varying degrees of success. Without therapy, the prognosis is good, but the inesthetics lesions persists forever. ${ }^{2}$ For our patient, we were have chosen excision methode. It's more interesting because it was diagnosis and therapeutic method.

\section{Conclusion}

Nevoid hyperkeratosis of the nipple and areola is a rare benign dermatologic condition with unknown etiology, clinical presentation may be difficult to distinguish from other pathologies of the nipple. Biopsy is essential to establish the diagnosis. It is mostly a cosmetic problem of young women. Specific treatment guidelines do not exist because of the unknown pathogenesis. ${ }^{11}$ Our observation is distinguished by its clinical and atypical dermoscopic appearance simulating seborrheic keratosis.

\section{Acknowledgements}

None.

\section{Conflict of interest}

Authors declare that there is no conflict of interest.

\section{References}

1. Ghanadan A, Balighi K, Khezri S, et al. Nevoid hyperkeratosis of the nipple and/or areola: Treatment with topical steroid. Indian J Dermatol. 2013;58(5):408

2. Reza Yaghoobi, Amir Feily. Bilateral nevoid hyperkeratosis of the nipples and areolae. International Journal of Dermatology. 2015;54(1):e47-e48.

3. Tocco-Tussardi I, Mobargha N, Bassetto F, et al. Radical treatment of extensive nevoid hyperkeratosis of the areola and breast with surgical excision after mild response to topical agents: A case report. Int $J$ Surg Case Rep. 2016;28:117-120.

4. Mazzella C, Costa C, Fabbrocini G, et al. Nevoid hyperkeratosis of the nipple mimicking a pigmented basal cell carcinoma. JAAD Case Reports. 2016;2(6):500-501.

5. Foustanos A, Panagiotopoulos K, Ahmad D, et al. Surgical Approach for Nevoid Hyperkeratosis of the Areola. J Cutan Aesthet Surg. 2012;5(1):4042.

6. Shastry V, Betkerur J, Kushalappa PA. Unilateral nevoid hyperkeratosis of the nipple: A report of two cases. Indian J Dermatol Venereol Leprol. 2006;72(4):303-305.

7. Chikhalkar SB, Misri R, Kharkar V. Nevoid hyperkeratosis of nipple: Nevoid or hormonal? Indian J Dermatol Venereo. 2006;72(5):384-386.

8. Sengul N, Parlak AH, Oruk S, et al. Nevoid hyperkeratosis of the nipple and areola: a diagnosis of exclusion. Breast J. 2006;12(4):383-384.

9. Fenniche $\mathrm{S}$, Badri T. Nevoid hyperkeratosis of the nipple andareola. $N$ Engl JMed. 2010;362:1618.

10. Baykal C, bûyûkbabani N, Kavak A, et al. Nevoid hyperkeratosis of the nipple and areola: a distinct entity. J Am Acad Dermatol. 2002;46(3):414 418.

11. Sengül N, Parlak AH, Oruk $S$, et al. Nevoid Hyperkeratosis of the Nipple and Areola: A Diagnosis of Exclusion. The Breast Journal. 2006;12(4):383-384. 